\title{
Search for long-lived neutral particles decaying into lepton-jets with the ATLAS detector in proton-proton collision data at $\sqrt{s}=13 \mathrm{TeV}$
}

\author{
Del Gaudio Michela, on behalf of the ATLAS Collaboration \\ Universitá della Calabria and INFN-Cosenza \\ E-mail: michela.del.gaudio@cern.ch
}

\begin{abstract}
Several models of elementary particle physics Beyond the Standard Model predict the existence of neutral particles that can also be long lived and decay in collimated jets of light leptons and hadrons (lepton-jets). The present contribution collects the results about the displaced leptonjet search with the ATLAS experiment at the proton-proton LHC collider at $\sqrt{s}=13 \mathrm{TeV}$ during the 2015 data taking period $\left(3.4 \mathrm{fb}^{-1}\right)$. The results are interpreted in terms of the FalkowskyRuderman-Volansky-Zupan models where dark photons are generated through the decay of a Higgs boson, produced via gluon-fusion. The selected events are compared with the Standard Model expectations and with Beyond Standard Model predictions.
\end{abstract}

EPS-HEP 2017, European Physical Society conference on High Energy Physics 5-12 July 2017

Venice, Italy 


\section{Motivation and Benchmark Models}

A wide variety of Beyond Standard Model (BSM) theories predict a hidden sector weakly coupled to the visible one, via vector portal, i.e, through a long lived dark photon $\left(\gamma_{d}\right)$ with mass in the $\mathrm{MeV}$ to $\mathrm{GeV}$ range, which mixes kinetically with the Standard Model (SM) photon [1].

New heavy particles, or even H/W/Z/t, produced at the LHC may decay to the hidden sector and some hidden particles like $\gamma_{d}$ may decay back to SM particles. If the $\gamma_{d}$ is the lightest unstable particle of this dark sector, it will decay mainly to leptons and light mesons. There are various mechanisms by which particles obtain significant lifetimes in BSM theories. For example the decay of such particles can be suppressed in the hidden sector models where barrier potentials reduce the rate of kinematically allowed decays. Since the $\gamma_{d}$ lifetime is given by the kinetic mixing parameter between SM and hidden sector, which ranges between $10^{-3}$ to $10^{-4}$, the $\gamma_{d}$ has a non-negligible lifetime. At the LHC, $\gamma_{d}$ would be produced with large boost, yielding highly-collimated decay products in the final state.

This motivates the search for "displaced lepton-jets" (dLJs): collimated jet-like structures, containing pair(s) of muons, electrons, and/or light hadrons, produced far from the primary vertex.

The Falkowski-Ruderman-Volansky-Zupan (FRVZ) models are used as benchmark for the search presented here. The Falkowski-Ruderman-Volansky-Zupan (FRVZ) models assume that a SM Higgs boson (or even a BSM heavy Higgs boson) finally decays to dLJs [2], as shown in Figure 1. The hidden fermion $f_{d 2}$ decays to a $\gamma_{d}$ and an Hidden Lightest Stable Particle (HLSP), and each $\gamma_{d}$ decays to a dLJ (Figure 1 left); the $f_{d 2}$ decays to an HLSP and an hidden scalar $s_{d 1}$ that in turn decays to pairs of $\gamma_{d}$ (Figure 1 right). In the FRVZ models the $\gamma_{d}$ branching ratio depends on its mass. If the mass is $400 \mathrm{MeV}$, the $\gamma_{d}$ decays for $45 \%$ in pairs of electrons or muons, and for $10 \%$ in pairs of pions[2]. The mean lifetime (expressed as $\tau$ times the speed of light $\mathrm{c}$ ) is a free parameter [3].
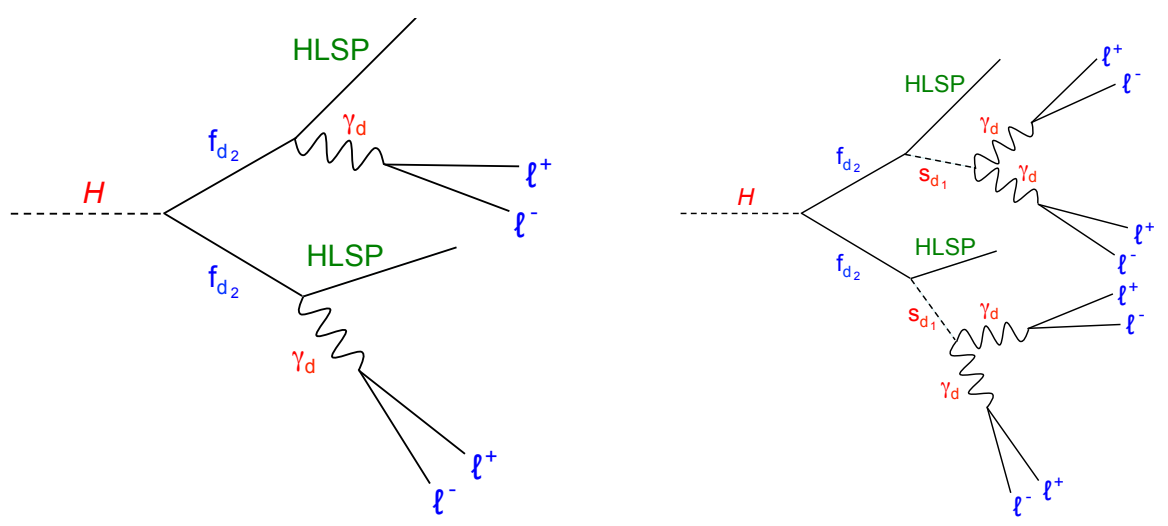

Figure 1: The FRVZ benchmark models: the diagrams show the production of hidden fermions and the their decay-chain in the hidden sector yielding two HLSP and $\gamma_{d}$ particles. In the FRVZ scenario, $\gamma_{d}$ are long lived particles, decaying in displaced and collimated jet-like structures.

\section{Lepton-jets reconstruction and selection}

This analysis only considers $\gamma_{d}$ decays that occur beyond the Pixel Detector and before the 
Muon Spectrometer [4]. Figure 2 shows the topologies of dLJs analyzed in this search:

- TYPE0: a cluster of at least two muons identified into the muon spectrometer with a veto on jets in a cone of radius $\Delta R=0.5$. It corresponds to at least one $\gamma_{d} \rightarrow \mu^{+} \mu^{-}$.

- TYPE1: a cluster of at least one jet and two muons in a cone of radius $\Delta R=0.5$. It corresponds to at least one $\gamma_{d} \rightarrow \mu^{+} \mu^{-}$and one $\gamma_{d} \rightarrow \pi^{+} \pi^{-}\left(e^{+} e^{-}\right)$.

- TYPE2: a jet with low electromagnetic fraction and no muons in a cone of radius $\Delta R=0.5$. It corresponds to at least one $\gamma_{d} \rightarrow \pi^{+} \pi^{-}\left(e^{+} e^{-}\right)$.
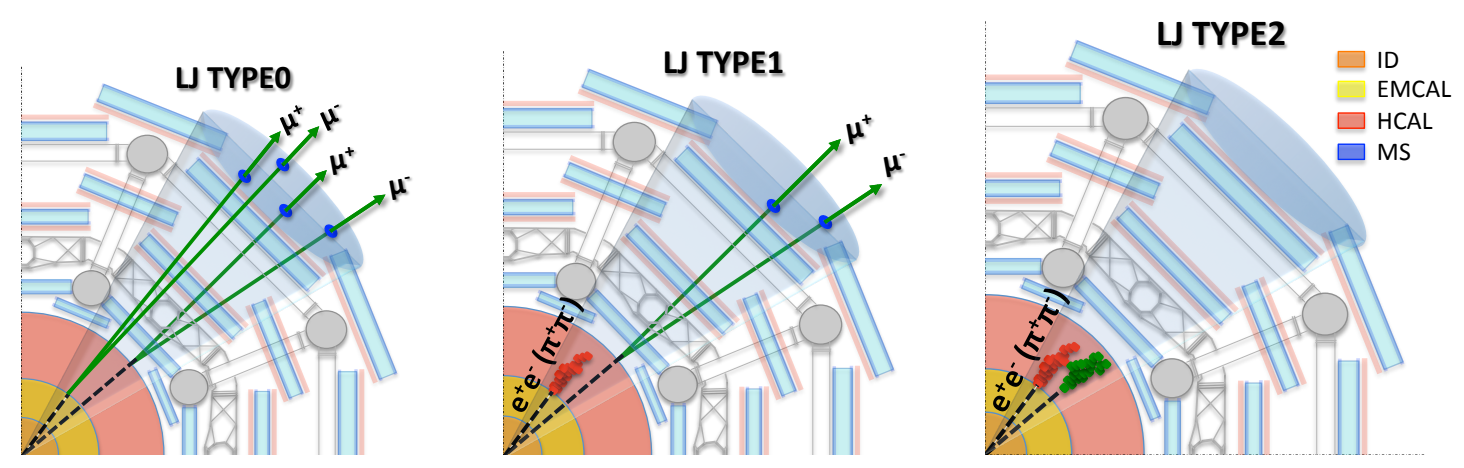

Figure 2: Classification of dLJs based on particle species content

Due to the dLJs definition as cluster of collimated particles, created far away from the primary vertex, this search is a challenge both for the reconstruction capability of the ATLAS detector, and also for the selection procedure. The triggers used to select dLJS of TYPE0 and TYPE1 are multi-muon triggers with muons reconstructed only in the muon spectrometer, instead for TYPE2 the single jet trigger with low electromagnetic fraction is used, therefore only dLJs produced in the hadronic calorimeters are selected.

To reduce the background, mainly constituted by muons from cosmic rays and QCD multi-jet event, selection requirements are used. To reduce the cosmic rays background are applied requirement on the jet timing and on the muon impact parameter, instead the QCD multi-jet background is reduced using track isolation in the inner tracker, jet electromagnetic fraction and width of the jets. Moreover, this search selects only events with two dLJs (in any of the possible combinations of TYPES), having an angular separation $|\Delta \varphi| \geq 1$. To evaluate the residual QCD multi-jet background and cosmics background a data-driven matrix $\mathrm{ABCD}$ method is used which assumes that the background is factorizable in two independent variables: $\Sigma p_{T}$ (scalar sum of the $p_{T}$ of ID tracks in a $\Delta R=0.5$ cone around the $\mathrm{dLJ}$ ) and $|\Delta \varphi|$ (angular separation between the two dLJs). The events of the FRVZ models, considered in this analysis, have high $|\Delta \varphi|$ and low $\Sigma p_{T}$.

\section{Results}

The observed yield is consistent with the expected background. The results are used to set 95\% CLs exclusion limits on $\sigma \times B R\left(H \rightarrow 2(4) \gamma_{d}+X\right)$ as a function of the $\gamma_{d}$ mean lifetime $\tau$ in 
the two FRVZ models. The Figure 3 shows the exclusion limits on the $\sigma \times B R$ for the $125 \mathrm{GeV}$ Higgs boson SM gluon-fusion production cross section. The dashed curve is the expected limit, the solid curve is the observed one and the horizontal lines correspond to $\sigma \times B R$ for two values of the BR of the Higgs boson decay to dark photons. Due the low signal efficiency for the TYPE2TYPE2 channel, for all the limit setting the TYPE2-TYPE2 events have been excluded. The same exclusion limits have been calculated for the $800 \mathrm{GeV}$ Higgs-like heavy scalar boson [3].
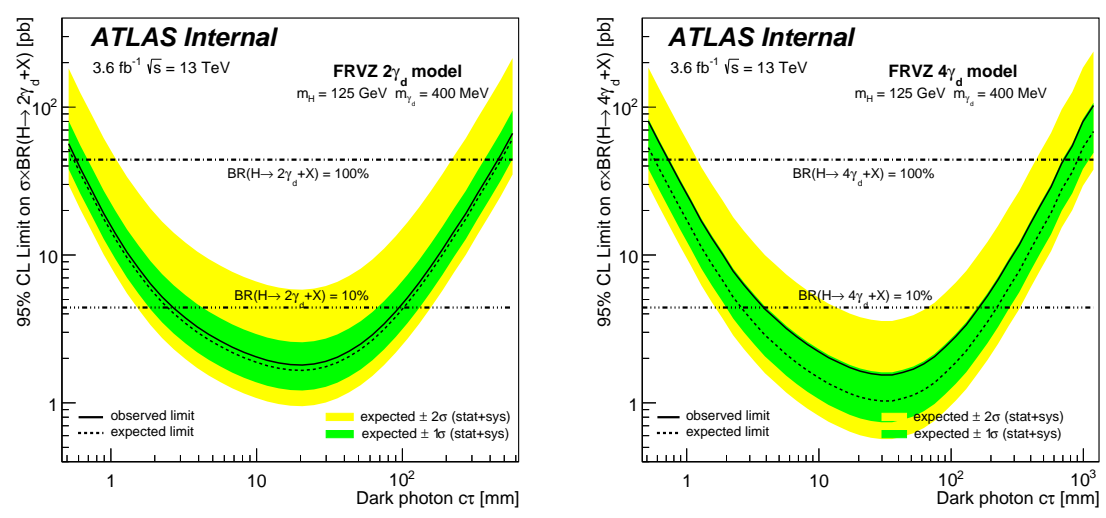

Figure 3: The 95\% upper limits on $\sigma \times B R\left(H \rightarrow 2 \gamma_{d}+X\right)$ (left) and on $\sigma \times B R\left(H \rightarrow 4 \gamma_{d}+X\right)$ (right) with the $125 \mathrm{GeV}$ Higgs boson, as a function of the $\gamma_{d}$ lifetime $\tau$. The horizontal lines are the values of $\sigma \times B R$ for the Higgs boson decay to dark photons. TYPE2-TYPE2 events are excluded [3].

Recent observation of anomalous internal pair creation in ${ }^{8} \mathrm{Be}$ is interpreted as a possible signature of a light, neutral boson of $16.7 \mathrm{MeV}$ decaying in $e^{-} e^{+}$pairs from the dark sector [5]. A TYPE2-TYPE2 signal can be related to the framework of this "protophobic" boson assuming that a $800 \mathrm{GeV}$ Higgs-like heavy scalar boson decays with a BR of $100 \%$ to $2 \gamma_{D}+X$. The $\gamma_{D}$ mass is assumed $16.7 \mathrm{MeV}$ and it decays only to electron pairs.

\section{References}

[1] Strassler M. J., et Zurek K. M., Echoes of a Hidden Valley at Hadron Colliders, Phys. Lett. B 651 (2007) 374

[2] Falkowski A., et Ruderman J. T., et Volansky T., et Zupan J., Hidden Higgs Decaying to Lepton Jets JHEP 1005, 077 (2010)

[3] ATLAS Collaboration Search for long-lived neutral particles decaying into displaced lepton-jets in proton-proton collisions at sqrts $=13 \mathrm{TeV}$ with the ATLAS detector, DOI:ATLAS-CONF-2016-042 (2016), https://cds.cern.ch/record/2206083

[4] ATLAS Collaboration, The ATLAS Experiment at the CERN Large Hadron Collider, JINST 3 (2008) S08003

[5] Krasznahorkay A. J., et al., Observation of Anomalous Internal Pair Creation in ${ }^{8}$ Be: A Possible Signature of a Light, Neutral Boson, Phys. Rev. Lett. 116042501 (2016) 